\title{
Machine Learning Overview
}

\author{
Taiwo Oladipupo Ayodele \\ University of Portsmouth \\ United Kingdom
}

\section{Machine Learning Overview}

Machine Learning according to Michie et al (D. Michie, 1994) is generally taken to encompass automatic computing procedures based on logical or binary operations that learn a task from a series of examples. Here we are just concerned with classification, and it is arguable what should come under the Machine Learning umbrella. Attention has focussed on decision-tree approaches, in which classification results from a sequence of logical steps. These are capable of representing the most complex problem given sufficient data (but this may mean an enormous amount!). Other techniques, such as genetic algorithms and inductive logic procedures (ILP), are currently under active development and in principle would allow us to deal with more general types of data, including cases where the number and type of attributes may vary, and where additional layers of learning are superimposed, with hierarchical structure of attributes and classes and so on. Machine Learning aims to generate classifying expressions simple enough to be understood easily by the human. They must mimic human reasoning sufficiently to provide insight into the decision process. Like statistical approaches, background knowledge may be exploited in development, but operation is assumed without human intervention.

To learn is:

- to gain knowledge, comprehension, or mastery of through experience or study or to gain knowledge (of something) or acquire skill in (some art or practice)

- to acquire experience of or an ability or a skill in

- to memorize (something), to gain by experience, example, or practice.

Machine Learning can be defines as a process of building computer systems that automatically improve with experience, and implement a learning process. Machine Learning can still be defined as learning the theory automatically from the data, through a process of inference, model fitting, or learning from examples:

- Automated extraction of useful information from a body of data by building good probabilistic models.

- Ideally suited for areas with lots of data in the absence of a general theory. 
A major focus of machine learning research is to automatically produce models and a model is a pattern, plan, representation, or description designed to show the main working of a system, or concept, such as rules determinate rule for performing a mathematical operation and obtaining a certain result, a function from sets of formulae to formulae, and patterns ( model which can be used to generate things or parts of a thing from data.

Learning is a MANY-FACETED PHENOMENON as described by Jaime et al (Jaime G. Carbonell, 1983) and also stated that Learning processes include the acquisition of new declarative knowledge, the development of motor and cognitive skills through instruction or practice, the organization of new knowledge into general, effective representations, and the discovery of new facts and theories through observation and experimentation. The study and computer modelling of learning processes in their multiple manifestations constitutes the subject matter of machine learning. Although machine learning has been a central concern in artificial intelligence since the early days when the idea of "self-organizing systems" was popular, the limitations inherent in the early neural network approaches led to a temporary decline in research volume. More recently, new symbolic methods and knowledge-intensive techniques have yielded promising results and these in turn have led to the current, revival in machine learning research. This book examines some basic methodological issues, existing techniques, proposes a classification of machine learning techniques, and provides a historical review of the major research directions.

Machine Learning according to Michie et al (D. Michie, 1994) is generally taken to encompass automatic computing procedures based on logical or binary operations that learn a task from a series of examples. Here we are just concerned with classification, and it is arguable what should come under the Machine Learning umbrella. Attention has focussed on decision-tree approaches, in which classification results from a sequence of logical steps. These are capable of representing the most complex problem given sufficient data (but this may mean an enormous amount!). Other techniques, such as genetic algorithms and inductive logic procedures (ILP), are currently under active development and in principle would allow us to deal with more general types of data, including cases where the number and type of attributes may vary, and where additional layers of learning are superimposed, with hierarchical structure of attributes and classes and so on. Machine Learning aims to generate classifying expressions simple enough to be understood easily by the human. They must mimic human reasoning sufficiently to provide insight into the decision process. Like statistical approaches, background knowledge may be exploited in development, but operation is assumed without human intervention. Machine learning has always been an integral part of artificial intelligence according to Jaime et al (Jaime G. Carbonell, 1983), and its methodology has evolved in concert, with the major concerns of the field. In response to the difficulties of encoding ever increasing volumes of knowledge in model AI systems, many researchers have recently turned their attention to machine learning as a means to overcome the knowledge acquisition bottleneck. This book presents a taxonomic analysis of machine learning organized primarily by learning strategies and secondarily by knowledge representation and application areas. A historical survey out lining the development of various approaches to machine learning is presented from early neural networks to present knowledge-intensive techniques. 


\subsection{The Aim of Machine Learning}

The field of machine learning can be organized around three primary research Areas:

- Task-Oriented Studies: The development and analysis of learning systems oriented toward solving a predetermined set, of tasks (also known as the "engineering approach").

- Cognitive Simulation: The investigation and computer simulation of human learning processes (also known as the "cognitive modelling approach")

- Theoretical Analysis: the theoretical exploration of the space of possible learning methods and algorithms independent application domain.

Although many research efforts strive primarily towards one of these objectives, progress in on objective often lends to progress in another. For example, in order to investigate the space of possible learning methods, a reasonable starting point may be to consider the only known example of robust learning behaviour, namely humans (and perhaps other biological systems) Similarly, psychological investigations of human learning may held by theoretical analysis that may suggest various possible learning models. The need to acquire a particular form of knowledge in stone task-oriented study may itself spawn new theoretical analysis or pose the question: "how do humans acquire this specific skill (or knowledge)?" The existence of these mutually supportive objectives reflects the entire field of artificial intelligence where expert system research, cognitive simulation, and theoretical studies provide some (cross-fertilization of problems and ideas (Jaime G. Carbonell, 1983).

\subsubsection{Applied Learning Systems}

At, present, instructing a computer or a computer-controlled robot, to perform a task requires one to define a complete and correct, algorithm for that task, and then laboriously program the algorithm into a computer. These activities typically involve a tedious and time-consuming effort by specially trained personnel. Present-day computer systems cannot truly learn to perform a task through examples or by analogy to a similar, previous-solved task. Nor can they improve significantly on the basis of past, mistakes or acquire new abilities by observing and imitating experts. Machine learning research strives to open the possibility of instructing computers in such new ways, and thereby promises to ease the burden of hand-programming growing volumes of increasingly complex information into the computers of tomorrow. The rapid expansion of application and availability of computers today makes this possibility even more attractive and desirable.

\subsubsection{Knowledge acquisition}

When approaching a task-oriented knowledge acquisition task, one must be aware that, the resultant computer system must interact with humans, and therefore should closely parallel human abilities. The traditional argument that an engineering approach need not reflect human or biological performance and is not, truly applicable to machine learning. Since airplane, a successful result on an almost pure engineering approach, better little resemblance to their biological counterparts, one may argue that applied knowledge 
acquisition systems could be equally divorced from any consideration of human capabilities. This argument does not apply

here because airplanes need not interact, with or understand birds Learning machines, on the other hand, will have to interact, with the people who make use of them, and consequently the concept and skills they acquire- if not necessarily their internal mechanism and must be understandable to human.

\subsection{Machine Learning as a Science}

The clear contender for a cognitive invariant in human is the learning mechanism which is the ability facts, skills and more abstractive concepts. Therefore understanding human learning well enough to reproduce aspect of that learning behaviour in a computer system is, in itself, a worthy scientific goal. Moreover, the computer can render substantial assistance to cognitive psychology, in that it may be used to test the consistency and completeness of learning theories and enforce a commitment to the fine-structure processlevel detail that precludes meaningless tautological or untestable theories (Bishop, 2006).

The study of human learning processes is also of considerable practical significance. Gaining insights into the principles underlying human learning abilities is likely to lead to more effective educational techniques. Machine learning research is all about developing intelligent computer assistant or a computer tutoring systems and many of these goals are shared within the machine learning fields. According to Jaime et al (Jaime G. Carbonell, 1983) who stated computer tutoring are starting to incorporate abilities to infer models of student competence from observed performance. Inferring the scope of a student's knowledge and skills in a particular area allows much more effective and individualized tutoring of the student (Sleeman, 1983).

The basic scientific objective of machine learning is the exploration of possible learning mechanisms, including the discovery of different induction algorithms, the scope of theoretical limitations of certain method seems to be the information that must be available to the learner, the issue of coping with imperfect training data and the creation of general techniques applicable in many task domains. There is not reason to believe that human learning methods are the only possible mean of acquiring knowledge and skills. In fact, common sense suggests that human learning represents just one point in an uncharted space of possible learning methods- a point that through the evolutionary process is particularly well suited to cope with the general physical environment in which we exist. Most theoretical work in machine learning are centred on creation, characterization and analysis of general learning methods, with the major emphasis on analyzing generality and performance rather than psychological plausibility.

Whereas theoretical analysis provides a means of exploring the space of possible learning methods, the task-oriented approach provides a vehicle to test and improve the performance of functional learning systems and testing applied learning systems, one can determine the cost-effectiveness trade-offs and limitations of particular approaches to learning. In this way, individual data points in the space possible learning systems are explored and the space itself becomes better understood.

Knowledge Acquisition and Skill Refinement: There are two basic form of learning:

1) Knowledge Acquisition

2) Skill refinement 
When it is said that someone learned mathematics, it means that this person acquired concepts of mathematics, understood the meaning and their relationship to each other as well as to the world. The importance of learning in this case is acquisition of knowledge, including the description and models of physical systems and their behaviours, incorporating a variety of representations from simple intrusive mental model models, examples and images to completely test mathematical equations and physical laws. A person is said to have learned more if this knowledge explains a broader scope of situations, is more accurate, and is better able to predict the behaviour of the typical world (Allix, 2003). This form of learning is typically to a large variety of situations and is generally learned knowledge acquisition. Therefore, knowledge acquisition is defined as learning a new task coupled with the ability to apply the information in the effective manner.

The second form of learning is the gradual improvement of motor and cognitive skills through practice- Learning by practice. Learning such as:

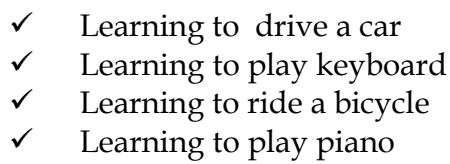

If one acquire all textbook knowledge on how to perform these aforementioned activities, this represent the initial phase in developing the required skills. So, the major part of the learning process consists of taming the acquired skill, and improving the mental or motor coordination or learning coordination by repeated practice and correction of deviations from desired behaviour. This form of learning often called skill taming. This differs in many ways from knowledge acquisition. Where knowledge acquisition may be a conscious process whose result is the creation of new representative knowledge structures and mental models, and skill taming is learning from example or learning from repeated practice without concerted conscious effort. Jamie (Jaime G. Carbonell, 1983) explained that most human learning appears to be a mixture of both activities, with intellectual endeavours favouring the former and motor coordination tasks favouring the latter. Present machine learning research focuses on the knowledge acquisition aspect, although some investigations, specifically those concerned with learning in problem-solving and transforming declarative instructions into effective actions, touch on aspects of both types of learning. Whereas knowledge acquisition clearly belongs in the realm of artificial intelligence research, a case could be made that skill refinement comes closer to non-symbolic processes such as those studied in adaptative control system. Hence, perhaps both forms of learning- (knowledge based and refinement learning) can be captured in artificial intelligence models.

\subsection{Classification of Machine Learning}

There are several areas of machine learning that could be exploited to solve the problems of email management and our approach implemented unsupervised machine learning method. Uunsupervised learning is a method of machine learning whereby the algorithm is presented with examples from the input space only and a model is fit to these observations. For example, a clustering algorithm would be a form of unsupervised learning. "Unsupervised learning is a method of machine learning where a model is fit to observations. It is distinguished from supervised learning by the fact that there is no $a$ priori 
output. In unsupervised learning, a data set of input objects is gathered. Unsupervised learning then typically treats input objects as a set of random variables. A joint density model is then built for the data set. The problem of unsupervised learning involved learning patterns in the input when no specific output values are supplied" according to Russell (Russell, 2003).

In the unsupervised learning problem, we observe only the features and have no measurements of the outcome. Our task is rather to describe how the data are organized or clustered". Hastie (Trevor Hastie, 2001) explained that "In unsupervised learning or clustering there is no explicit teacher, and the system forms clusters or "natural groupings" of the input patterns. "Natural" is always defined explicitly or implicitly in the clustering system itself; and given a particular set of patterns or cost function, different clustering algorithms lead to different clusters. Often the user will set the hypothesized number of different clusters ahead of time, but how should this be done? How do we avoid inappropriate representations?" according to Duda (Richard O. Duda, 2000).

There are various categories in the field of artificial intelligence. The classifications of machine learning systems are:

- Supervised Machine Learning: Supervised learning is a machine learning technique for learning a function from training data. The training data consist of pairs of input objects (typically vectors), and desired outputs. The output of the function can be a continuous value (called regression), or can predict a class label of the input object (called classification). The task of the supervised learner is to predict the value of the function for any valid input object after having seen a number of training examples (i.e. pairs of input and target output). To achieve this, the learner has to generalize from the presented data to unseen situations in a "reasonable" way (see inductive bias). (Compare with unsupervised learning.)

Supervised learning is a machine learning technique whereby the algorithm is first presented with training data which consists of examples which include both the inputs and the desired outputs; thus enabling it to learn a function. The learner should then be able to generalize from the presented data to unseen examples." by Mitchell (Mitchell, 2006). Supervised learning also implies we are given a training set of (X, Y) pairs by a "teacher". We know (sometimes only approximately) the values of $f$ for the $\mathrm{m}$ samples in the training set, $\equiv$ we assume that if we can find a hypothesis, $h$, that closely agrees with $f$ for the members of $\equiv$ then this hypothesis will be a good guess for $f$ especially if $\equiv$ is large. Curvefitting is a simple example of supervised learning of a function. Suppose we are given the values of a two-dimensional function. $f$, at the four sample points shown by the solid circles in Figure 9. We want to fit these four points with a function, $h$, drawn from the set, $H$, of second-degree functions. We show there a two-dimensional parabolic surface above the $x_{1} . x_{2}$, plane that fits the points. This parabolic function, $h$, is our hypothesis about the function $f$, which produced the four samples. In this case, $h=\mathrm{f}$ at the four samples, but we need not have required exact matches. Read more in section 3.1. 
- Unsupervised Machine Learning: Unsupervised learning ${ }^{1}$ is a type of machine learning where manual labels of inputs are not used. It is distinguished from supervised learning approaches which learn how to perform a task, such as classification or regression, using a set of human prepared examples. .Unsupervised learning means we are only given the Xs and some (ultimate) feedback function on our performance. We simply have a training set of vectors without function values of them. The problem in this case, typically, is to partition the training set into subsets, $\equiv_{1} \ldots \equiv_{R}$, in some appropriate way.

\subsubsection{Classification of Machine Learning}

Classification of machine learning system could be implemented along many different dimensions and we have chosen these two dimensions:

- Inference Learning: This is a form of classification on the basis of underlying strategy that is involved. These strategies will depend on the amount of inference the learning system performs on the information provided to the system.

Now learning strategies are distinguished by the amount of inference the learner performs on the information provided. So, if a computer system performs email classification for example, it knowledge increases but this may not perform any inference on the new information, this means all cognitive efforts is on the part of the analyst or programmer. But if the machine learning classifier independently discovers new theories or adopt new concepts, this will perform a very substantial inference. This is what is called deriving knowledge from example or experiments or by observation. An example is: When a student wants to solve statistical problems in a text book - this is a process that involves inference but the solution is not to discover a brand new formula without guidance from a teacher or text book. So, as the amount of inference that the learner is capable of performing increases, the burdens placed on the teacher or on external environ decreases. According to Jaime (Jaime G. Carbonell, 1983) , (Anil Mathur, 1999) who stated that it is much more difficult to teach a person by explaining each steps in a complex task than by showing that person the way that similar tasks are usually done. It more difficult yet to programme a computer to perform a complex task than to instruct a person to perform the task; as programming requires explicit specification of all prerequisite details, whereas a person receiving instruction can use prior knowledge and common sense to fill in most mundane details.

- Knowledge Representation: This is a form of skill acquire by the learner on the basis of the type of representation of the knowledge.

\subsubsection{Existing Learning Systems}

There are many other existing learning systems that employ multiple strategies and knowledge representations and some have been applied to more than one. In the knowledge based machine learning method, no inference is used but the learner display the transformation of knowledge in varieties of ways:

\footnotetext{
${ }^{1}$ http://en.wikipedia.org/wiki/Unsupervised_learning
} 
- Learning by being programmed: When an algorithm or code is written to perform specific task. E.g. a code is written as a guessing game for the type of animal. Such a programme could be modified by external entity.

- Learning by memorisation: This is by memorising given facts or data with no inference drawn from the incoming information or data.

- Learning from examples: This is a special case of inductive learning. Given a set of examples and counterexamples of a concept, the learner induces a general concept description that describes all of the positive examples and none of the counterexamples. Learning from examples is a method has been heavily investigated in artificial intelligence field. The amount of inference perform by the learner is much greater than in learning from instructions, (Anil Mathur, 1999), (Jaime G. Carbonell, 1983).

- Learning from Observation: This is an unsupervised learning approach and is a very general form of inductive learning that includes discovery systems, theory formation tasks, the creation of classification criteria to form taxonomic hierarchies and similar task to be performed without benefit of an external teacher. Unsupervised learning requires the learner to perform more inference than any approach as previously explained. The learner is not provided with a set if data or instance of a particular concept. The above classification of learning strategies should help one to compare various learning systems in terms of their underlying mechanisms, in terms of the available external source of information and in terms of the degree to which they reply on preorganised knowledge. Read more in section 3.2.

\subsection{Machine Learning Applications}

The other aspect for classifying learning systems is the area of application which gives a new dimension for machine learning. Below are areas to which various existing learning systems have been applied. They are:
1) Computer Programming
2) Game playing (chess, poker, and so on)
3) Image recognition, Speech recognition
4) Medical diagnosis
5) Agriculture, Physics
6) Email management, Robotics
7) Music
8) Mathematics
9) Natural Language Processing and many more.

\section{References}

Allix, N. M. (2003, April). Epistemology And Knowledge Management Concepts And Practices. Journal of Knowledge Management Practice .

Alpaydin, E. (2004). Introduction to Machine Learning. Massachusetts, USA: MIT Press.

Anderson, J. R. (1995). Learning and Memory. Wiley, New York, USA. 
Anil Mathur, G. P. (1999). Socialization influences on preparation for later life. Journal of Marketing Practice: Applied Marketing Science , 5 (6,7,8), 163 - 176.

Ashby, W. R. (1960). Design of a Brain, The Origin of Adaptive Behaviour. John Wiley and Son.

Batista, G. \&. (2003). An Analysis of Four Missing Data Treatment Methods for Suppervised Learning. Applied Artificial Intelligence, 17, 519-533.

Bishop, C. M. (1995). Neural Networks for Pattern Recognition. Oxford, England: Oxford University Press.

Bishop, C. M. (2006). Pattern Recognition and Machine Learning (Information Science and Statistics). New York, New York: Springer Science and Business Media.

Block H, D. (1961). The Perceptron: A Model of Brian Functioning. 34 (1), 123-135.

Carling, A. (1992). Introducing Neural Networks . Wilmslow, UK: Sigma Press.

D. Michie, D. J. (1994). Machine Learning, Neural and Statistical Classification. Prentice Hall Inc.

Fausett, L. (19994). Fundamentals of Neural Networks. New York: Prentice Hall.

Forsyth, R. S. (1990). The strange story of the Perceptron. Artificial Intelligence Review , 4 (2), 147-155.

Friedberg, R. M. (1958). A learning machine: Part, 1. IBM Journal , 2-13.

Ghahramani, Z. (2008). Unsupervised learning algorithms are designed to extract structure from data. 178 , pp. 1-8. IOS Press.

Gillies, D. (1996). Artificial Intelligence and Scientific Method. OUP Oxford.

Haykin, S. (19994). Neural Networks: A Comprehensive Foundation. New York: Macmillan Publishing.

Hodge, V. A. (2004). A Survey of Outlier Detection Methodologies. Artificial Intelligence Review, $22(2), 85-126$.

Holland, J. (1980). Adaptive Algorithms for Discovering and Using General Patterns in Growing Knowledge Bases Policy Analysis and Information Systems. 4 (3).

Hunt, E. B. (1966). Experiment in Induction.

Ian H. Witten, E. F. (2005). Data Mining Practical Machine Learning and Techniques (Second edition ed.). Morgan Kaufmann.

Jaime G. Carbonell, R. S. (1983). Machine Learning: A Historical and Methodological Analysis. Association for the Advancement of Artificial Intelligence, 4 (3), 1-10.

Kohonen, T. (1997). Self-Organizating Maps.

Luis Gonz, 1. A. (2005). Unified dual for bi-class SVM approaches. Pattern Recognition , 38 (10), 1772-1774.

McCulloch, W. S. (1943). A logical calculus of the ideas immanent in nervous activity. Bull. Math. Biophysics , 115-133.

Mitchell, T. M. (2006). The Discipline of Machine Learning. Machine Learning Department technical report CMU-ML-06-108, Carnegie Mellon University.

Mooney, R. J. (2000). Learning Language in Logic. In L. N. Science, Learning for Semantic Interpretation: Scaling Up without Dumbing Down (pp. 219-234). Springer Berlin / Heidelberg.

Mostow, D. (1983). Transforming declarative advice into effective procedures: a heuristic search cxamplc In I?. S. Michalski, Tioga Press.

Nilsson, N. J. (1982). Principles of Artificial Intelligence (Symbolic Computation / Artificial Intelligence). Springer.

Oltean, M. (2005). Evolving Evolutionary Algorithms Using Linear Genetic Programming. 13 (3), $387-410$. 
Orlitsky, A., Santhanam, N., Viswanathan, K., \& Zhang, J. (2005). Convergence of profile based estimators. Proceedings of International Symposium on Information Theory. Proceedings. International Symposium on, pp. 1843 - 1847. Adelaide, Australia: IEEE.

Patterson, D. (19996). Artificial Neural Networks. Singapore: Prentice Hall.

R. S. Michalski, T. J. (1983). Learning from Observation: Conceptual Clustering. TIOGA Publishing Co.

Rajesh P. N. Rao, B. A. (2002). Probabilistic Models of the Brain. MIT Press.

Rashevsky, N. (1948). Mathematical Biophysics:Physico-Mathematical Foundations of Biology. Chicago: Univ. of Chicago Press.

Richard O. Duda, P. E. (2000). Pattern Classification (2nd Edition ed.).

Richard S. Sutton, A. G. (1998). Reinforcement Learning. MIT Press.

Ripley, B. (1996). Pattern Recognition and Neural Networks. Cambridge University Press.

Rosenblatt, F. (1958). The perceptron: a probabilistic model for information storage and organization in the brain . Psychological Review , 65 (6), 386-408.

Russell, S. J. (2003). Artificial Intelligence: A Modern Approach (2nd Edition ed.). Upper Saddle River, NJ, NJ, USA: Prentice Hall.

Ryszard S. Michalski, J. G. (1955). Machine Learning: An Artificial Intelligence Approach (Volume I). Morgan Kaufmann .

Ryszard S. Michalski, J. G. (1955). Machine Learning: An Artificial Intelligence Approach.

Selfridge, O. G. (1959). Pandemonium: a paradigm for learning. In The mechanisation of thought processes. H.M.S.O., London. London.

Sleeman, D. H. (1983). Inferring Student Models for Intelligent CAI. Machine Learning. Tioga Press.

Tapas Kanungo, D. M. (2002). A local search approximation algorithm for k-means clustering. Proceedings of the eighteenth annual symposium on Computational geometry (pp. 10-18). Barcelona, Spain : ACM Press.

Timothy Jason Shepard, P. J. (1998). Decision Fusion Using a Multi-Linear Classifier . In Proceedings of the International Conference on Multisource-Multisensor Information Fusion.

Tom, M. (1997). Machibe Learning. Machine Learning, Tom Mitchell, McGraw Hill, 1997: McGraw Hill.

Trevor Hastie, R. T. (2001). The Elements of Statistical Learning. New york, NY, USA: Springer Science and Business Media.

Widrow, B. W. (2007). Adaptive Inverse Control: A Signal Processing Approach. Wiley-IEEE Press.

Y. Chali, S. R. (2009). Complex Question Answering: Unsupervised Learning Approaches and Experiments. Journal of Artificial Intelligent Research , 1-47.

Yu, L. L. (2004, October). Efficient feature Selection via Analysis of Relevance and Redundacy. JMLR , 1205-1224.

Zhang, S. Z. (2002). Data Preparation for Data Mining. Applied Artificial Intelligence. 17, 375 381. 


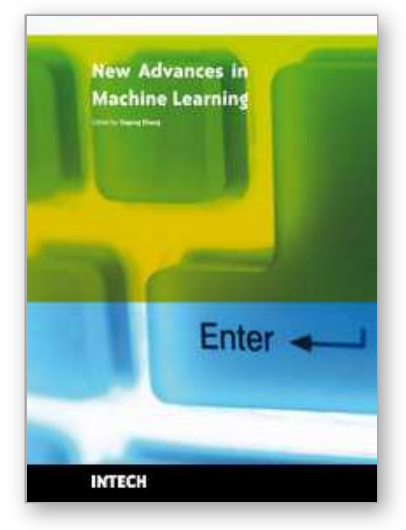

\author{
New Advances in Machine Learning \\ Edited by Yagang Zhang
}

ISBN 978-953-307-034-6

Hard cover, 366 pages

Publisher InTech

Published online 01, February, 2010

Published in print edition February, 2010

The purpose of this book is to provide an up-to-date and systematical introduction to the principles and algorithms of machine learning. The definition of learning is broad enough to include most tasks that we commonly call "learning" tasks, as we use the word in daily life. It is also broad enough to encompass computers that improve from experience in quite straightforward ways. The book will be of interest to industrial engineers and scientists as well as academics who wish to pursue machine learning. The book is intended for both graduate and postgraduate students in fields such as computer science, cybernetics, system sciences, engineering, statistics, and social sciences, and as a reference for software professionals and practitioners. The wide scope of the book provides a good introduction to many approaches of machine learning, and it is also the source of useful bibliographical information.

\title{
How to reference
}

In order to correctly reference this scholarly work, feel free to copy and paste the following:

Taiwo Oladipupo Ayodele (2010). Machine Learning Overview, New Advances in Machine Learning, Yagang Zhang (Ed.), ISBN: 978-953-307-034-6, InTech, Available from: http://www.intechopen.com/books/newadvances-in-machine-learning/machine-learning-overview

\section{INTECH}

open science | open minds

\author{
InTech Europe \\ University Campus STeP Ri \\ Slavka Krautzeka 83/A \\ 51000 Rijeka, Croatia \\ Phone: +385 (51) 770447 \\ Fax: +385 (51) 686166 \\ www.intechopen.com
}

\author{
InTech China \\ Unit 405, Office Block, Hotel Equatorial Shanghai \\ No.65, Yan An Road (West), Shanghai, 200040, China \\ 中国上海市延安西路65号上海国际贵都大饭店办公楼405单元 \\ Phone: +86-21-62489820 \\ Fax: $+86-21-62489821$
}


(C) 2010 The Author(s). Licensee IntechOpen. This chapter is distributed under the terms of the Creative Commons Attribution-NonCommercialShareAlike-3.0 License, which permits use, distribution and reproduction for non-commercial purposes, provided the original is properly cited and derivative works building on this content are distributed under the same license. 